\title{
Near-IR Imaging and Optical Spectroscopy of M 2-9
}

\section{A. Arrieta and S. Torres-Peimbert}

Instituto de Astronomía, UNAM

IR OBSERVATIONS. We have extended previous infrared imaging studies (cf. Hora \& Latter 1994 ApJ 437, 281; Kastner et al. 1996 ApJ 462, 777) through wide and narrow band filters.

In these images: (a) $J H K^{\prime}$ morphology is similar to the optical structure. The knots are brighter at the shorter wavelengths, while the core is brighter at $K^{\prime}$. (b) [He I] 1.083 $\mu \mathrm{m}$, that traces the highly ionized region, is concentrated along the axial region and extends throughout the lobes (33" $\times 9$ "). It shows the knots very prominently. (c) [Fe II] $1.644 \mu \mathrm{m}$, that traces the shock excited regions, extends []throughout the lobes; it is more prominent in the northern knot and it is very strong in the core. (d) The excited molecular $\mathrm{H}_{2}$ lines are concentrated on a wide 'torus' in the equatorial plane. This geometry is similar to that exhibited by most bipolar planetary nebulae where $\mathrm{H}_{2}$ has been detected (e.g., Kastner et al. 1996). No $\mathrm{H}_{2}$ emission is seen in the core. (e) Pa $\gamma$ shows emission in only in the core and the northern knot. (f) $\mathrm{Br} \gamma$ shows emission in the core and in knots N2, S2 and S3. (g) No significant emission in CO was obtained.

HIGH DISPERSION OPTICAL OBSERVATIONS. Long slit echelle spectroscopy was obtained from 3600 to $6800 \AA$. From this we analyze the lobes and the central region.

Lobes The emission spectrum is dominated by the $\mathrm{H}$ Balmer lines; they also show emission in $\mathrm{He} \mathrm{I}$, [N II], [N I], [O III], [O II], [O I], [S III], and [S II]. (a) In all emission lines the southern lobe emission is blueshifted while the northern one is redshifted relative to the systemic velocity (typically $\Delta \mathrm{V} \sim 30 \mathrm{~km} / \mathrm{s}$ between the bright knots separated $\sim$ 40"). (b) The condensations appear as intensity maxima with peculiar velocities relative to the systemic velocity.

Core The core shows a faint continuum without appreciable absorption features and a very complex emission spectrum. In addition to those lines characteristic of $\mathrm{PNe}$, it exhibits numerous relatively bright emission lines ([Fe II], [Fe III], Si II, [Fe I] and [Ni II]). These lines are also prominent in $\eta$ Car and in RR Tel. All emission lines are very wide, with a FWHM from 50 to $100 \mathrm{~km} \mathrm{~s}^{-1},-\mathrm{H} \alpha$ has been reported to have extensions up to $1000 \mathrm{~km} \mathrm{~s}^{-1}$, typical of symbiotic stars (Balick 1989, AJ 97, 671). The velocity profiles of the core region show that there are different unresolved regions at different densities and with large velocities that are producing the emission. The Balmer lines are double peaked with a dominating red component, some high excitation forbidden lines are double peaked with a dominating it blue component ([O III], [Ne III], ...) while others are single peaked (He I 5876, [N II] 6584, [O I] 6300) at the mean velocity.

The red component is produced at $N_{e}>10^{6} \mathrm{~cm}^{-3}$, while the blue component is produced at lower densities $\left(10^{6}>N_{e}>10^{4} \mathrm{~cm}^{-3}\right)$. We interpret this as evidence of dense gas surrounding a possible binary system, either: $(i)$ a rotating disk with a high density receding section and a low density approaching section, or (ii) a high density rotating disk and a low density nebular condensation ejected toward us.

The observations were carried out at the 2-m telescope of Observatorio Astronómico Nacional in San Pedro Mártir, Baja California, México. 